\section{Azithromycin-Nonsusceptible Shigella flexneri 3a in Men Who Have Sex with Men, Taiwan, 2015-2016}

\section{Ying-Shu Liao, Yen-Yi Liu, Yi-Chun Lo, Chien-Shun Chiou}

Author affiliation: Taiwan Centers for Disease Control, Taipei, Taiwan

DOI: http://dx.doi.org/10.3201/eid2302.161260

We report an outbreak of azithromycin-nonsusceptible Shigella flexneri 3a infection in Taiwan associated with men who have sex with men. The bacterial strains belonged to the sublineage $A$ of a recently reported outbreak lineage associated with men who have sex with men, characterized by reduced azithromycin susceptibility and circulation in shigellosis low-risk regions.

Chigellosis among men who have sex with men (MSM)

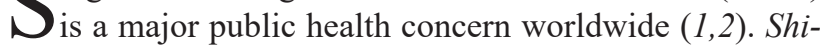
gella flexneri serotype 3 a was responsible for a prolonged MSM-associated outbreak in England and Wales initially detected in 2009 and ongoing at the time of study publication (3). More recently, S. flexneri 3a strains with a genetic lineage distinct from Africa- and Asia-associated lineages were identified among MSM in Europe, Australia, and North America (4). All strains in this lineage carried a mobile element with 4 antimicrobial resistance genes (blaoxA-1, catAl, aadAl, and tetB), and most recently emerging strains harbored a conjugative IncFII plasmid pKSR100, which conferred high-level resistance to azithromycin (4).

During 2005-2014, the incidence of domestically acquired shigellosis in Taiwan was low $(0.7 / 100,000$ persons) (5). Shigellosis is reported through the Web-based National Notifiable Disease Surveillance System (NDSS), which has been in operation since 1997 to monitor all notifiable diseases in Taiwan $(6,7)$. The NDSS detected the first shigellosis outbreak in MSM during March-May 2015; the outbreak was determined to be caused by the global spread of a ciprofloxacin-resistant $S$. sonnei clone (8). During June 2015-May 2016, a total of 200 shigellosis cases were reported to the NDSS, of which 21 were domestically acquired $S$. flexneri 3a infections in northern and central Taiwan. All 21 of the $S$. flexneri 3a infections were in men 22-44 years of age, including 17 self-reported MSM. Of the 21 case-patients, most had reported other infections before $S$. flexneri 3a diagnosis: HIV $(\mathrm{n}=16), 0-162$ (median 49) months earlier; syphilis $(\mathrm{n}=17), 0-85$ (median 9.5) months earlier; gonorrhea $(n=6), 14-130$ (median 26) months earlier; amebiasis $(n=2), 0-112$ (median 56) months earlier; acute hepatitis A ( $\mathrm{n}=1), 0$ months earlier; and $S$. sonnei infection $(\mathrm{n}=1), 3$ months earlier.

We tested the $21 \mathrm{~S}$. flexneri $3 \mathrm{a}$ isolates for antimicrobial resistance by using a custom-made 96-well Sensititer MIC panel (Trek Diagnostic Systems Ltd., West Grinstead, UK) and the Etest kit (bioMérieux, Marcy l'Etoile, France). All 21 were resistant to ampicillin, chloramphenicol, streptomycin, and tetracycline, and 19 were nonsusceptible to azithromycin (MIC 64-96 $\mu \mathrm{g} / \mathrm{mL}$ ).

We also used the PulseNet pulsed-field gel electrophoresis (PFGE) protocol to analyze the isolates (9) and compared the PFGE patterns with those of 30 S. flexneri $3 \mathrm{a}$ isolates isolated in Taiwan during 2000-2011. The NotI digested PFGE patterns of 20/21 of the isolates were identical to the earlier isolates, and the remaining isolate showed a highly similar pattern. Clustering analysis of the PFGE patterns by using BioNumerics version 6.6 software (Applied Maths, Sint-Martens-Latem, Belgium) revealed that the 21 isolates were genetically distant from the 2000-2011 $S$. flexneri 3a isolates.

We used the Illumina MiSeq platform (Illumina, San Diego, CA, USA) for whole-genome sequencing of 4 isolates, of which 3 were azithromycin-nonsusceptible (codes R15.1162, R16.001, and R16.0013) and 1 was azithromycin-susceptible (R15.3406). We deposited the raw sequence reads in the National Center for Biotechnology Information Short Read Archive database (accession no. SRP080176; BioProject PRJNA335684). We used CLC Genomics Workbench version 9.0.1 (CLC bio, Aarhus, Denmark) for de novo assembly of the reads. We uploaded contigs for each isolate to the website of Center for Genome Epidemiology (http://www.genomicepidemiology.org/) and used its tools to identify sequence type (Multi Locus Sequnce Typing toolkit), plasmid type (PlasmidFinder), and antimicrobial resistance genes (ResFinder 2.1).

The 4 isolates were sequence type 245 (ST245), harbored plasmids belonging to incompatibility groups IncFII and Col, and had resistance genes bla ${ }_{\text {OXA-1 }}$, catAl, aadA1, tetB, ermB, mphA, and $b l a_{\mathrm{TEM}}$. Although isolate R15.3406 carried an intact $m p h A$ gene, it was phenotypically azithromycin susceptible. We compared genome single-nucleotide polymorphisms and constructed trees by using the CSI Phylogeny pipeline (https://cge.cbs.dtu.dk/services/CSIPhylogeny/) (10) to infer phylogenetic relationships between the 4 isolates and the 331 strains used in the study by Baker et al. (4). These analyses showed that the 4 isolates recovered in Taiwan belonged to the sublineage A of the MSMassociated outbreak lineage reported by Baker et al. (4).

In conclusion, we report an azithromycin-nonsusceptible S. flexneri 3a outbreak associated with MSM in Taiwan. The 21 isolates were genetically distant from the $S$. flexneri 3a isolates recovered in Taiwan from 2000-2011 and belonged to the sublineage $A$ of the recently reported 
MSM-associated outbreak lineage characterized by reduced azithromycin susceptibility and circulation in shigellosis low-risk regions (4). The introduction of this MSM-associated S. flexneri 3a lineage into Taiwan in 2015 illustrates that the pathogen can spread rapidly across continents, possibly through intensified sexual networks among MSM $(2,8)$. We recommend continued surveillance for antimicrobial resistance genes in S. flexneri to inform clinical management of shigellosis among MSM and public health interventions where needed, including appropriate antimicrobial drug stewardship.

\section{Acknowledgments}

This study was funded by the Ministry of Health and Welfare, Taiwan (grant no. MOHW105-CDC-C-315-123301).

Ms. Liao is a senior researcher in the Centers for Disease Control, Taiwan. Her research interests include molecular subtyping methods in the epidemiology of foodborne bacterial disease.

\section{References}

1. Daskalakis DC, Blaser MJ. Another perfect storm: Shigella, men who have sex with men, and HIV. Clin Infect Dis. 2007;44:335-7. http://dx.doi.org/10.1086/510591

2. Simms I, Field N, Jenkins C, Childs T, Gilbart VL, Dallman TJ, et al. Intensified shigellosis epidemic associated with sexual transmission in men who have sex with men-Shigella flexneri and S. sonnei in England, 2004 to end of February 2015. Euro Surveill. 2015;20:21097. http://dx.doi.org/10.2807/1560-7917. ES2015.20.15.21097

3. Borg ML, Modi A, Tostmann A, Gobin M, Cartwright J, Quigley C, et al. Ongoing outbreak of Shigella flexneri serotype 3a in men who have sex with men in England and Wales, data from 2009-2011. Euro Surveill. 2012;17:2-6.

4. Baker KS, Dallman TJ, Ashton PM, Day M, Hughes G, Crook PD, et al. Intercontinental dissemination of azithromycin-resistant shigellosis through sexual transmission: a cross-sectional study. Lancet Infect Dis. 2015;15:913-21. http://dx.doi.org/10.1016/ S1473-3099(15)00002-X

5. Taiwan Centers for Disease Control. Taipei: Taiwan National Infectious Disease Statistics System [cited 2016 Nov 2]. http://nidss.cdc.gov.tw/en/Default.aspx

6. Lo YC, Ji DD, Hung CC. Prevalent and incident HIV diagnoses among Entamoeba histolytica-infected adult males: a changing epidemiology associated with sexual transmission-Taiwan, 2006-2013. PLoS Negl Trop Dis. 2014;8:e3222. http://dx.doi.org/ 10.1371/journal.pntd.0003222

7. Lo YC, Tsai MS, Sun HY, Hung CC, Chuang JH. National trend and characteristics of acute hepatitis C among HIV-infected individuals: a matched case-control study-Taiwan, 2001-2014. PLoS One. 2015;10:e0139687. http://dx.doi.org/10.1371/journal. pone. 0139687

8. Chiou CS, Izumiya H, Kawamura M, Liao YS, Su YS, Wu HH, et al. The worldwide spread of ciprofloxacin-resistant Shigella sonnei among HIV-infected men who have sex with men, Taiwan. Clin Microbiol Infect. 2016;22:383.e11-6. http://dx.doi/org/ 10.1016/j.cmi.2015.12.021

9. Ribot EM, Fair MA, Gautom R, Cameron DN, Hunter SB, Swaminathan B, et al. Standardization of pulsed-field gel electrophoresis protocols for the subtyping of Escherichia coli O157:H7, Salmonella, and Shigella for PulseNet.

Foodborne Pathog Dis. 2006;3:59-67. http://dx.doi.org/10.1089/ fpd.2006.3.59

10. Kaas RS, Leekitcharoenphon P, Aarestrup FM, Lund O. Solving the problem of comparing whole bacterial genomes across different sequencing platforms. PLoS One. 2014;9:e104984. http://dx.doi.org/10.1371/journal.pone.0104984

Address for correspondence: Chien-Shun Chiou, Central Regional Laboratory, Center for Diagnostics and Vaccine Development, Centers for Disease Control, Taichung 40855, Taiwan; email: nipmcsc@cdc.gov.tw

\section{Fatal Emmonsia sp. Infection and Fungemia after Orthotopic Liver Transplantation}

\author{
Shanthi Kappagoda, Jason Y. Adams, \\ Robert Luo, Niaz Banaei, Waldo Concepcion, \\ Dora Y. Ho
}

Author affiliations: Stanford University School of Medicine, Stanford, California, USA (S. Kappagoda, R. Luo, N. Banaei, W, Concepcion, D.Y. Ho); University of California, Davis, California, USA (J.Y. Adams)

DOI: http: http://dx.doi.org/10.3201/eid2302.160799

We report a fatal case of disseminated Emmonsia sp. infection in a 55-year-old man who received an orthotopic liver transplant. The patient had pneumonia and fungemia, and multisystem organ failure developed. As human habitats and the number of immunocompromised patients increase, physicians must be aware of this emerging fungal infection.

$E$ mmonsia species are ubiquitous, soil-dwelling saprophytic fungi. Two species, E. crescens and E. parva, cause pulmonary disease (adiaspiromycosis) in rodents and other small animals. After inhalation, the conidia (adiaspores) grow without replication or dissemination and can cause pulmonary granulomas. Human cases are rare and usually occur in immunocompetent hosts $(1,2)$. However, disseminated infections caused by $E$. pasteuriana-like species have been reported primarily in HIV-infected patients in South Africa $(3,4)$. A recent review implicated novel Emmonsia spp.-like fungi as emerging agents of disseminated infection (1). We report a case of fatal disseminated infection after orthotopic liver transplantation caused by a novel Emmonsia sp. 